\title{
Article \\ Effect of the Osteotomy Inclination Angle in the Sagittal Plane on the Posterior Tibial Slope of the Tibiofemoral Joint in Medial Open-Wedge High Tibial Osteotomy: Three-Dimensional Computed Tomography Analysis
}

\author{
Jai Hyun Chung ${ }^{1}$, Chong Hyuk Choi ${ }^{2}$, Sung-Hwan Kim ${ }^{2}$, Sung-Jae Kim ${ }^{2}$, Seung-Kyu Lee ${ }^{3}$ and Min Jung ${ }^{2, *(1)}$ \\ 1 Department of Medicine, Yonsei University Graduate School, Seoul 03722, Korea; jjhsky0843@gmail.com \\ 2 Arthroscopy and Joint Research Institute, Department of Orthopaedic Surgery, Yonsei University College of \\ Medicine, Seoul 03722, Korea; choi8422@yuhs.ac (C.H.C.); orthohwan@yuhs.ac (S.-H.K.); \\ severanscopy@yuhs.ac (S.-J.K.) \\ 3 Department of Orthopaedic Surgery, Yonsei University College of Medicine, Seoul 03722, Korea; \\ terry1376@yuhs.ac \\ * Correspondence: jmin1103@yuhs.ac; Tel.: +82-2-2228-2184
}

Citation: Chung, J.H.; Choi, C.H.; Kim, S.-H.; Kim, S.-J.; Lee, S.-K.; Jung, M. Effect of the Osteotomy Inclination Angle in the Sagittal Plane on the Posterior Tibial Slope of the Tibiofemoral Joint in Medial Open-Wedge High Tibial Osteotomy: Three-Dimensional Computed Tomography Analysis. J. Clin. Med. 2021, 10, 4272. https://doi.org/ $10.3390 /$ jcm 10184272

Academic Editor: Emmanuel Andrès

Received: 22 July 2021

Accepted: 18 September 2021

Published: 21 September 2021

Publisher's Note: MDPI stays neutral with regard to jurisdictional claims in published maps and institutional affiliations.

Copyright: (c) 2021 by the authors. Licensee MDPI, Basel, Switzerland. This article is an open access article distributed under the terms and conditions of the Creative Commons Attribution (CC BY) license (https:/ / creativecommons.org/licenses/by/ $4.0 /)$.
Abstract: The posterior tibial slope of the tibiofemoral joint changes after medial open wedge high tibial osteotomy (MOWHTO), but little is known about the effect of the sagittal osteotomy inclination angle on the change in the posterior tibial slope of the tibiofemoral joint. The purpose of this study was to investigate the effect of the osteotomy inclination angle in the sagittal plane on changes in the posterior tibial slope after MOWHTO by comparing how anterior and posterior inclination affect the posterior tibial slope of the tibiofemoral joint. The correlation between the osteotomy inclination angle and the postoperative posterior tibial slope angle was also assessed. Between May 2011 and November 2017, 80 patients with medial compartment osteoarthritis who underwent MOWHTO were included. The patients were divided into two groups according to the sagittal osteotomy inclination angle on the 3D reconstructed model. patients with an osteotomy line inclined anteriorly to the medial tibial plateau line were classified into group A (58 patients). patients with posteriorly inclined osteotomy line were classified as group P (22 patients). In the 3D reconstructed model, the preoperative and postoperative posterior tibial slope, osteotomy inclination angle relative to medial tibial plateau line in sagittal plane, and gap distance and ratio of the anterior and posterior osteotomy openings were measured. The preoperative and postoperative hip-knee-ankle angle, weight-bearing line ratio, and posterior tibial slope were also measured using plain radiographs. In the $3 \mathrm{D}$ reconstructed model, the postoperative posterior tibial slope significantly increased in group A (preoperative value $=9.7 \pm 2.9^{\circ}$, postoperative value $=10.7 \pm 3.0^{\circ}, p<0.001$ ) and decreased in group $\mathrm{P}$ (preoperative value $=8.7 \pm 2.7^{\circ}$, postoperative value $=7.7 \pm 2.7^{\circ}, p<0.001$ ). The postoperative posterior tibial slope (group $\mathrm{A}=10.7 \pm 3.0^{\circ}$, group $\mathrm{P}=7.7 \pm 2.7^{\circ}, p<0.001$ ) and posterior tibial slope change before and after surgery (group $\mathrm{A}=1.0 \pm 0.8^{\circ}$, group $\mathrm{P}=-0.9 \pm 0.8^{\circ}$, $p<0.001)$ also differed significantly between the groups. The Pearson correlation coefficient was $0.875(p<0.001)$ for the osteotomy inclination angle, and multivariate regression analysis showed that the only significant factor among the variables was the sagittal osteotomy inclination angle ( $\beta$ coefficient $=0.216, p<0.001$ ). The posterior tibial slope changed according to the osteotomy inclination angle in the sagittal plane after MOWHTO. The postoperative posterior tibial slope tended to increase when the osteotomy line was inclined anteriorly with respect to the medial tibial plateau line but decreased when the osteotomy line was inclined posteriorly. To avoid inadvertent change of posterior tibial slope, close attention needs to be paid to maintaining the sagittal osteotomy line parallel to the medial joint line during MOWHTO.

Keywords: osteoarthritis; high tibial osteotomy; posterior tibial slope; inclination angle 


\section{Introduction}

Medial open wedge high tibial osteotomy (MOWHTO) is an established, effective operative treatment for relatively young and active patients with osteoarthritis in the medial compartment of the tibiofemoral joint and varus deformity [1,2]. MOWHTO reduces the pressure of the medial compartment in the tibiofemoral joint by realigning the mechanical axis from the medial to the lateral side in the coronal plane [3,4]. This procedure has been reported to have good long-term clinical outcomes such as reduced pain and improved knee joint function [5-7]. Previous studies on MOWHTO tended to focus mainly on the change of alignment in the coronal plane regarding correction angle and postoperative coronal realignment [8-10]. However, MOWHTO is the three-dimensional (3D) surgical procedure. Therefore, the postoperative effect of osteotomy on the proximal tibia should also be considered in the sagittal plane. In addition to correction in the coronal plane, maintaining alignment in the sagittal plane has been reported to be important. The change in the posterior tibial slope in the sagittal plane influences the biomechanics of the knee joint. The increase in posterior tibial slope of the tibiofemoral joint leads to anterior translation of the tibia relative to the femur, thus developing an overload on the anterior cruciate ligament [11,12]. An increased posterior tibial slope of the tibiofemoral joint could also cause a redistribution of pressure into the posterior tibia in the anterior cruciate ligamentdeficient knee, resulting in degenerative change in articular cartilage [13] and an increase of vertical vector force onto the patellofemoral joint, which could lead to osteoarthritis of the patellofemoral joint $[14,15]$.

Despite efforts to maintain the posterior tibial slope of the tibiofemoral joint after MOWHTO, the posterior tibial slope tends to change after MOWHTO $[12,16]$. Change in posterior tibial slope before and after MOWHTO can be affected by various factors, including the osteotomy opening gap ratio between the anterior and posterior cortex $[17,18]$. Recently, the inclination angle of osteotomy in the sagittal plane has also been reported to affect change in the posterior tibial slope [19]. However, to the best of our knowledge, there has been no comprehensive study on the effect of the osteotomy inclination angle in the sagittal plane on changes in the posterior tibial slope after MOWHTO, including the correlation between the osteotomy inclination angle and postoperative posterior tibial slope angle. Therefore, the purpose of this study was to investigate the effect of the osteotomy inclination angle in the sagittal plane on the change in posterior tibial slope after MOWHTO by comparing how anterior and posterior inclination affect the posterior tibial slope and assessing the correlation between the osteotomy inclination angle and postoperative posterior tibial slope angle. The measurement of values in this study was performed not only in two-dimensional radiographs, but also in 3D reconstructed images. It was hypothesized that anterior or posterior inclination of osteotomy in the sagittal plane would influence the change in the posterior tibial slope after MOWHTO.

\section{Materials and Methods}

\subsection{Study Participants}

After approval by the institutional review board of our institution, patients with medial compartment osteoarthritis who underwent MOWHTO between May 2011 and November 2017 were retrospectively reviewed. A total of 169 knees were treated with MOWHTO during the study period. patients who met the following criteria, including the surgical indications of MOWHTO, were included in the study: (1) symptomatic medial compartment osteoarthritis with Kellgren-Lawrence grade III or more, (2) varus deformity of the lower extremity $>5^{\circ}$, (3) preserved cartilage in the lateral compartment (International Cartilage Repair Society [ICRS] grade [20] I or II), and (4) age of $<65$ years. patients with the following criteria were excluded from the study: (1) patients who did not have a postoperative computed tomography (CT) scan for the full length of the tibia and reconstructed 3D model; (2) a history of previous surgery on the affected knee; and (3) the presence of ligament injury on the affected knee. After applying the inclusion and exclusion criteria, 80 knees were included in this study. The patients were divided into two groups according 
to the osteotomy inclination angle in the sagittal plane in the 3D model reconstructed CT scan images. When the osteotomy line was inclined anteriorly with respect to the medial tibial plateau line, the cases were classified into group A (Figure 1A), while when the osteotomy line was inclined posteriorly with respect to the medial tibial plateau line, the cases were classified into group P (Figure 1B). group A and group P consisted of 58 and 22 patients, respectively.

A

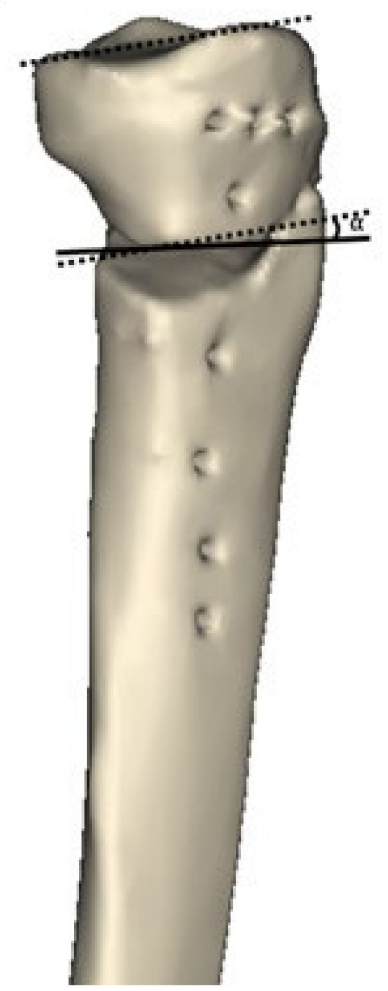

B

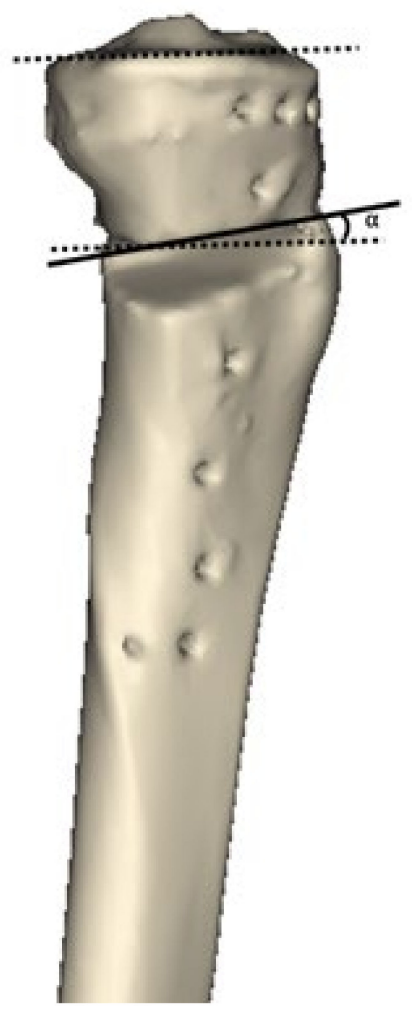

Figure 1. The patients were divided into two groups according to the osteotomy inclination angle in the sagittal plane in a three-dimensional reconstructed model. On the true lateral view, a black dotted line was drawn as a medial tibial plateau line. A black solid line was drawn as a sagittal osteotomy line on the anteromedial aspect of the lowermost part of the proximal tibial segment. The angle $\left(\alpha^{\circ}\right)$ formed by these two lines was defined as the osteotomy inclination angle. (A) group A, the osteotomy line is inclined anteriorly with respect to the medial tibial plateau line. (B) group $\mathrm{P}$, the osteotomy line is inclined posteriorly with respect to the medial tibial plateau line.

\subsection{Operative Procedure}

The preoperative plan was made with a double-limb standing antero-posterior radiograph of the full-length lower extremity. The Miniaci method was used to obtain the valgus correction target angle [21]. The realigned weight-bearing line was aimed at passing the Fujisawa point (62.5\% of the tibial plateau from the medial edge) from the center of the femoral head [22]. Arthroscopy was performed before the osteotomy. Thereafter, an oblique skin incision was made on the anteromedial aspect of the proximal tibia, and the superficial medial collateral ligament was released. Two Kirschner wires were inserted from the upper border of the pes anserinus to the fibular head under the guidance of an image intensifier. Primary transverse osteotomy was conducted along these two Kirschner wires, and a secondary ascending osteotomy was performed posterior to the tibial tuberosity. After gradually opening the osteotomy site with four chisels, it was opened with a bone spreader up to the planned correction angle. The ratio of the anterior gap to the posterior gap of the opening was maintained at approximately 2 to $3[17,18,23]$. A TomoFix plate 
(DePuy Synthes, West Chester, PA, USA) was fixed with locking screws to the proximal tibia to maintain the osteotomy gap.

\subsection{Reconstruction of 3D Computed Tomography Model}

A postoperative CT scan was taken on the day of the operation. CT evaluations were performed using the CT scanner Sensation 64 (Siemens Healthcare, Erlangen, Germany). The tube parameters were $120 \mathrm{kVp}$ and $135 \sim 253 \mathrm{mAs}$. The acquisition matrix was $512 \times 512$ pixels. The scan field of view was $134 \sim 271 \mathrm{~mm}$, and the slice thickness was $0.6 \sim 1 \mathrm{~mm}$. A CT scan was done with the knee in full extension. Digital Imaging and Communications in Medicine (DICOM) data were downloaded from the picture archiving and communication system (Centricity PACS, GE Medical System Information Technologies, Milwaukee, Wisconsin). The axial, coronal, and sagittal DICOM images were then imported into Mimics software (version 17; Materialise, Leuven, Belgium), and a 3D bone model of the femur and tibia treated with MOWHTO was reconstructed. The plate and locking screws were removed digitally from the 3D model, leaving only the tibia after osteotomy.

\subsection{Measurement of Variables on 3D Reconstructed Model}

Three major variables were measured in the 3D reconstructed model: (1) the preoperative and postoperative posterior tibial slope, (2) osteotomy inclination angle relative to the medial tibial plateau line in the sagittal plane, and (3) anterior and posterior opening gap and the ratio of these two osteotomy gaps. To measure the posterior tibial slope and osteotomy inclination angle in the sagittal plane, the 3D reconstructed model was aligned in a true lateral position. The true lateral position of the tibia was obtained by manipulating the femoral lateral and medial condyles to be superimposed on the basis of the method presented in a previous study [24]. When measuring variables, the 3D model of the femur was made invisible for convenience. After the true lateral view was completed, this view was captured because these two lines could not be located on the same plane. The posterior tibial slope was measured on the captured 3D true lateral view as previously described $[25,26]$. The line perpendicular to the bisecting line of the tibial shaft and the medial tibial plateau line were drawn. The angle formed by these two lines was defined as the posterior tibial slope (Figure 2A). To measure the preoperative original posterior tibial slope, the original tibia model was obtained by removing the osteotomy gap of the proximal tibia. The proximal and distal parts of the tibia were combined to the preoperative original tibia by rotating the proximal segment using the lateral cortex as a hinge. The original posterior tibial slope was measured in the same way (Figure 2B). To measure the osteotomy inclination angle in the sagittal plane, a medial tibial plateau line and a sagittal osteotomy line on the anteromedial aspect of the lowermost part of the proximal tibia segment were drawn on the captured 3D true lateral view. The angle formed by these two lines was defined as the osteotomy inclination angle (Figure 3A) [19]. When the front of the osteotomy line was inclined downward with respect to the line parallel to the medial tibial plateau, it was classified as positive, and if it was inclined upward, it was classified as negative. The osteotomy gap ratio was defined as the ratio between the distances of the anterior opening gap and posterior opening gap in the 3D reconstructed model. The anterior opening gap was measured at the medial edge of the frontal plane osteotomy site. The posterior opening gap was measured at the most prominent posteromedial edge of the tibia (Figure 3B) $[17,27]$. The osteotomy gap ratio (\%) was calculated by dividing the anterior opening gap by the posterior opening gap and multiplying by 100 . Measurements of variables were conducted by two independent orthopedic surgeons who did not participate in the MOWHTO and were blinded to the patients' information to increase their reliability. The mean of the two numerical values was used. 
A

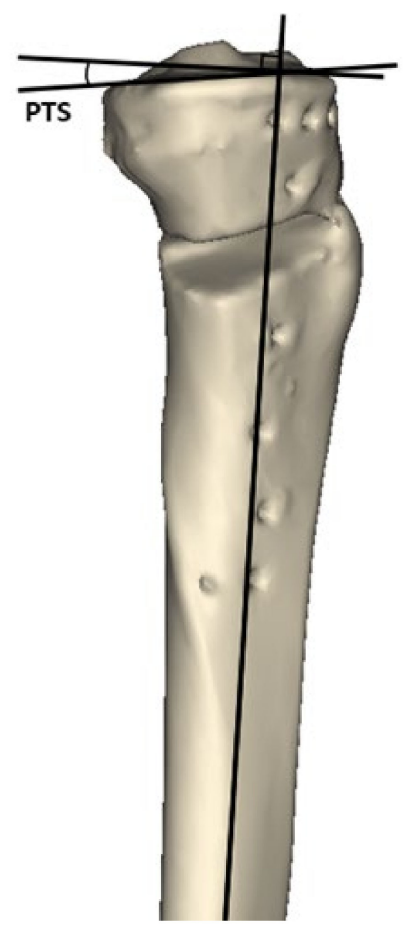

B

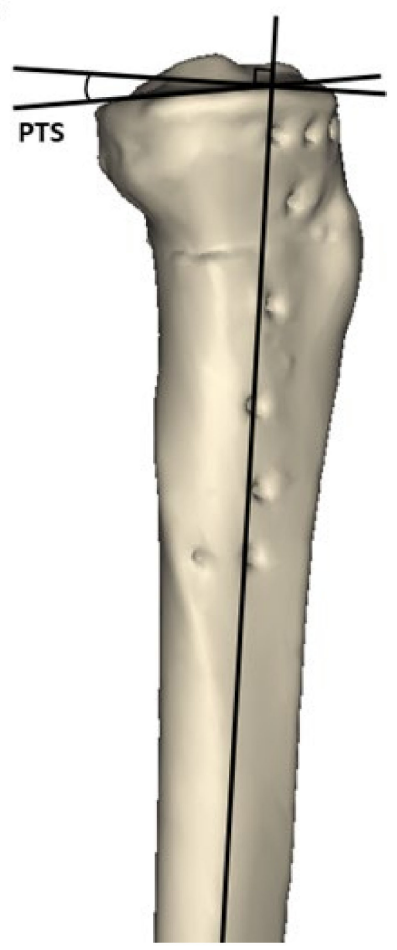

Figure 2. Measurement of the posterior tibial slope in a three-dimensional reconstructed model. The line perpendicular to the bisecting line of the tibial shaft and medial tibial plateau line was drawn on a true lateral view. The angle formed by these two lines was defined as the posterior tibial slope. (A) Postoperative posterior tibial slope. (B) Preoperative posterior tibial slope measured in the restored original preoperative tibia model. posterior tibial slope $=$ posterior tibial slope.

A

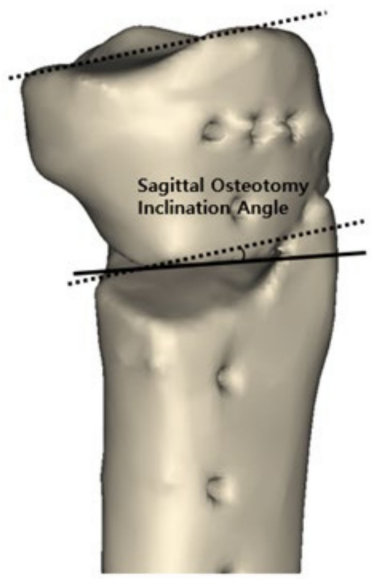

B

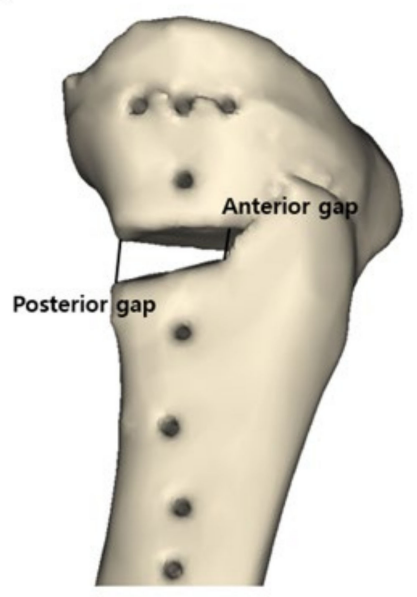

Figure 3. Measurement of the sagittal osteotomy inclination angle and anterior and posterior opening gap in a three-dimensional reconstructed model. (A) The sagittal osteotomy inclination angle was measured as the angle formed by the medial tibial plateau line (black dotted line) and osteotomy line on the anteromedial aspect of the lowermost part of the proximal tibial segment (black solid line) on the true lateral view. (B) Distances of anterior opening gap and posterior opening gap were measured in the three-dimensional reconstructed model. The anterior opening gap was measured at the medial edge of the frontal plane osteotomy site. The posterior opening gap was measured at the most prominent posteromedial edge of tibia. 


\subsection{Measurement on Plain Radiographs}

In addition to the measurement of variables in the 3D reconstructed model, the preoperative and postoperative hip-knee-ankle angle, weight-bearing line ratio, and posterior tibial slope were also measured using plain radiographs. Plain knee radiographs of the anteroposterior and lateral views were taken preoperatively and postoperatively on the day of the operation. Full-length lower extremity standing radiographs were also taken preoperatively and 6 months postoperatively. The hip-knee-ankle angle was measured on the full-length lower extremity standing radiograph with the first line from the femoral head center to the tibial spine center and the second line from the tibial spine center to the center of the superior articular surface of the talus. The angle made by the intersection of these two lines was defined as the hip-knee-ankle angle (Figure 4A) [28]. The weightbearing line ratio was measured with the weight-bearing line and tibial plateau line. The weight-bearing line was drawn from the center of the femoral head to the middle point of the superior articular surface of the talus on the full-length lower extremity standing radiograph. A tibial plateau line from the medial edge to the lateral edge of the proximal tibia on the joint surface was drawn thereafter. The weight-bearing line ratio was calculated as the ratio of the distance from the medial edge of the proximal tibial plateau to the intersection of the weight-bearing line and the distance from the proximal tibial plateau line to the entire length of the proximal tibial plateau line. The medial tibial edge was $0 \%$, and the lateral tibial edge was $100 \%$ (Figure 4B) [28]. Posterior tibial slope was also measured on the lateral view of the plain radiograph taken in a true lateral position [29]. The dots were marked $7 \mathrm{~cm}$ and $12 \mathrm{~cm}$ below the joint line on the anterior and posterior cortex of the tibia. Two lines connecting the two dots marked at $7 \mathrm{~cm}$ and $12 \mathrm{~cm}$ were drawn, and a line passing through the midpoint of these two lines was defined as the tibial shaft axis. The posterior tibial slope was measured as the angle formed by the line perpendicular to the tibial shaft axis and medial tibial plateau line (Figure $4 \mathrm{C}$ ). These variables were also measured by two independent orthopedic surgeons. Measurements on the plain radiographs were made without knowing the measured values in the $3 \mathrm{D}$ model for each patient. The mean of the two numerical values was used.

\subsection{Statistical Analysis}

The normality of distribution test was assessed using the Kolmogorov-Smirnov test. After confirming that the data followed a normal distribution, an independent t-test was employed for continuous variables. A paired t-test was used to compare preoperative and postoperative variables. For categorical variables, the chi-square or Fisher's exact test was used. Pearson correlation analysis was used to evaluate the correlation between variables (inclination angle, gap ratio, and correction angle) and the change in posterior tibial slope. Subsequently, multiple regression analysis was conducted to estimate the effect of the independent variable (inclination angle, gap ratio, and correction angle) on the dependent variable (change in posterior tibial slope). The intraclass correlation coefficient was calculated to assess the interobserver reliabilities of each measurement. The level of significance was set at $p<0.05$. Statistical analyses were conducted using IBM SPSS Statistics for Windows software program (version 25.0; IBM, Armonk, NY, USA). The statistical power was calculated using $G^{*}$ Power (v 3.1) [30]. 


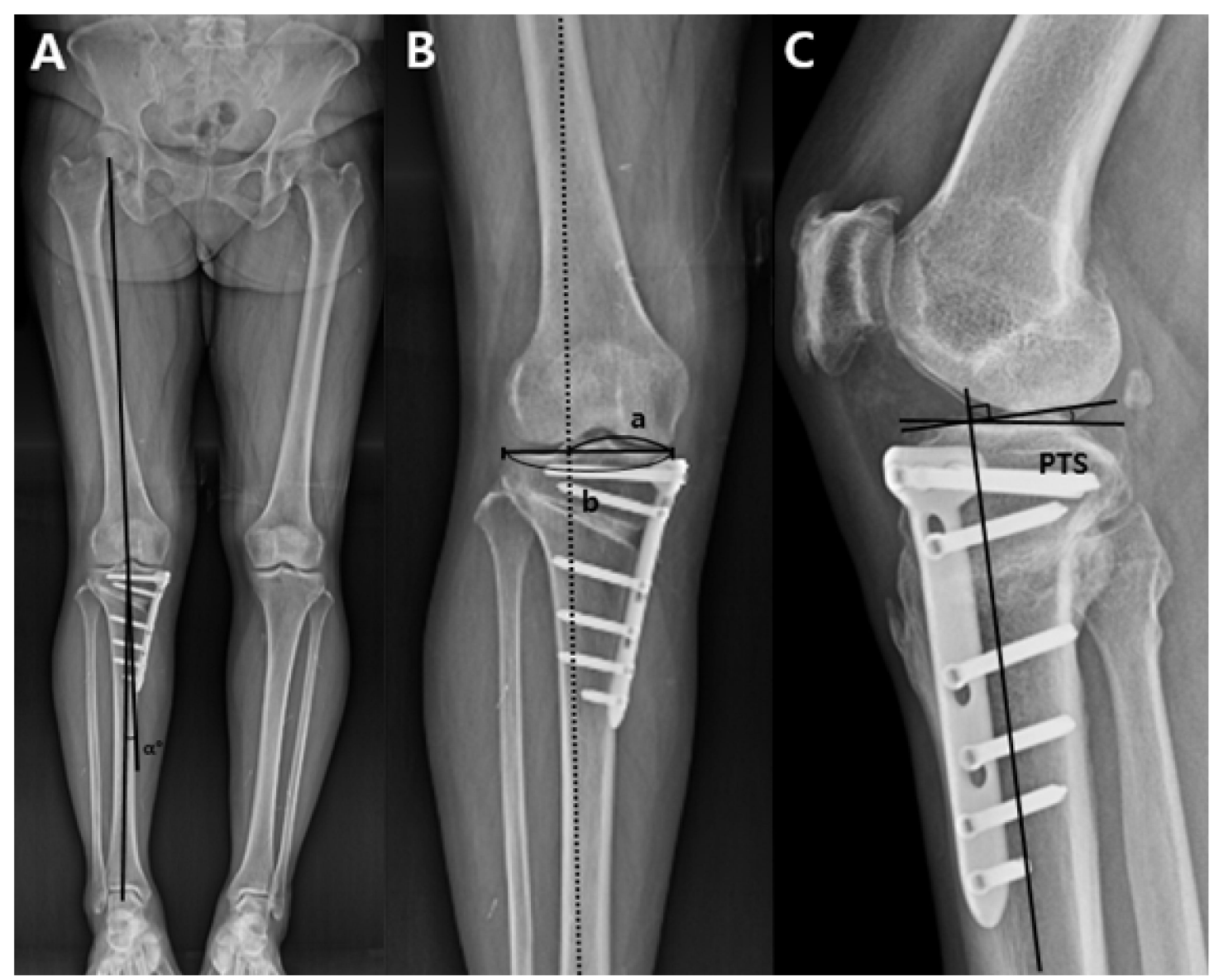

Figure 4. Measurement of the hip-knee-ankle angle, weight-bearing line ratio, and posterior tibial slope on the plain radiographs. (A) The hip-knee-ankle angle $\left(\alpha^{\circ}\right)$ is formed by the first line drawn from the center of the femoral center to center of the tibial spine and the second line drawn from the center of the tibial spine of knee joint to the center of the superior articular surface of the talus in the ankle joint. The angle made by the intersection of these two lines was defined as the hip-knee-ankle angle. (B) The weight-bearing line was drawn from the center of the femoral head to the middle point of the superior articular surface of the talus on the full-length lower extremity standing radiograph. A tibial plateau line from the medial edge to the lateral edge of the proximal tibia on the joint surface was drawn thereafter. Weight-bearing line ratio $(a / b)$ was calculated as the ratio of the distance from the medial edge of the proximal tibial plateau to the intersection of the weight-bearing line and the proximal tibial plateau line (a) to the entire length of the proximal tibial plateau line (b). The medial tibial edge was $0 \%$, and the lateral tibial edge was $100 \%$. (C) The posterior tibial slope is formed by the perpendicular line to the tibial shaft axis and the medial tibial plateau line on the true lateral view. posterior tibial slope $=$ posterior tibial slope.

\section{Results}

\subsection{Subjects}

The mean age at the time of surgery was 55.6 years in group A and 56.1 years in group P. There were 14 male and 44 female patients in group A and eight male and 14 female patients in group P. There was no statistically significant difference between the groups regarding age at the time of surgery, sex, affected side, and body mass index $(p>0.05)$ (Table 1).

\subsection{Measurement with Plain Radiographs}

On radiologic evaluation with plain radiographs, the preoperative values of hipknee-ankle angle (group $\mathrm{A}=-7.2 \pm 2.3^{\circ}$, group $\mathrm{P}=-7.8 \pm 2.8^{\circ}, p=0.319$ ), weightbearing line ratio (group $\mathrm{A}=19.3 \pm 8.5 \%$, group $\mathrm{P}=18.0 \pm 10.6 \%, p=0.343$ ), and posterior tibial slope (group $\mathrm{A}=9.5 \pm 3.1^{\circ}$, group $\mathrm{P}=8.3 \pm 2.7^{\circ}, p=0.117$ ) did not differ significantly between the groups. The postoperative values of hip-knee-ankle an- 
gle (group $\mathrm{A}=3.2 \pm 2.3^{\circ}$, group $\mathrm{P}=3.9 \pm 2.3^{\circ}, p=0.772$ ) and weight-bearing line ratio (group $\mathrm{A}=61.1 \pm 6.7 \%$, group $\mathrm{P}=63.9 \pm 7.2 \%, p=0.716$ ) did not differ significantly between the groups. The correction angle did not differ significantly between the groups: group $\mathrm{A}=11.0 \pm 2.5^{\circ}$; group $\mathrm{P}=11.6 \pm 2.6^{\circ}(p=0.396)$. However, the postoperative posterior tibial slope (group $\mathrm{A}=10.9 \pm 4.0^{\circ}$, group $\mathrm{P}=7.6 \pm 3.2^{\circ}, p=0.001$ ) and change in posterior tibial slope between the preoperative and postoperative values (group $\mathrm{A}=1.4 \pm 2.6^{\circ}$, group $\mathrm{P}=-0.8 \pm 2.3^{\circ}, p=0.001$ ) differed significantly between the groups (Table 2). A significant difference was also found in the comparison of posterior tibial slope before and after surgery for all patients (preoperative value $=9.1 \pm 3.1^{\circ}$, postoperative value $=9.8 \pm 4.1^{\circ}, p=0.008$ ). The preoperative and postoperative values within each group were significantly different only in group A (group A: preoperative value $=9.5 \pm 3.1^{\circ}$, postoperative value $=10.9 \pm 4.0^{\circ}, p<0.001$; group P: preoperative value $=8.3 \pm 2.7^{\circ}$, postoperative value $=7.6 \pm 3.2^{\circ}, p=0.137$ ) (Table 3 ). The intraclass correlation coefficients for interobserver reliability on plain radiograph were 0.748 (95\% confidence interval (CI), 0.607 0.838) for the preoperative posterior tibial slope and 0.759 (95\% CI, 0.625 0.846) for the postoperative posterior tibial slope. The intraclass correlation coefficients for hip-knee-ankle angle and weight-bearing line ratio are shown in Appendix A.

Table 1. Patient's demographic data.

\begin{tabular}{cccc}
\hline Variable & group A $(\boldsymbol{n}=\mathbf{5 8})$ & group P $(\boldsymbol{n}=\mathbf{2 2})$ & $\boldsymbol{p}$-Value \\
\hline Age * (years) & $55.6 \pm 5.3$ & $56.1 \pm 3.4$ & 0.648 \\
Sex + & 14 & 8 & 0.274 \\
Male & 44 & 14 & 0.617 \\
Female & & 12 & \\
Affected side + & 28 & 10 & 0.845 \\
Right & 30 & $27.0 \pm 2.7$ & \\
Left & $27.1 \pm 3.5$ & & \\
Body mass index $*\left(\mathrm{~kg} / \mathrm{m}^{2}\right)$ & & & \\
\hline
\end{tabular}

* Values are given as the mean \pm standard deviation.; $\uparrow$ Values are given as $n$.

Table 2. Preoperative and postoperative measurement on the plain radiograph.

\begin{tabular}{cccc}
\hline Variable & group A $(\boldsymbol{n}=\mathbf{5 8})$ & group P $(\boldsymbol{n}=\mathbf{2 2})$ & $\boldsymbol{p}$-Value \\
\hline Hip-knee-ankle angle $\left(^{\circ}\right)$ & & & \\
Preoperative value & $-7.2 \pm 2.3$ & $-7.8 \pm 2.8$ & 0.319 \\
Postoperative value & $3.2 \pm 2.3$ & $3.9 \pm 2.3$ & 0.772 \\
Weight-bearing line ratio $(\%)$ & & & \\
Preoperative value & $19.3 \pm 8.5$ & $18.0 \pm 10.6$ & 0.343 \\
Postoperative value & $61.1 \pm 6.7$ & $63.9 \pm 7.2$ & 0.716 \\
Correction angle $\left(^{\circ}\right)$ & $11.0 \pm 2.5$ & $11.6 \pm 2.6$ & 0.396 \\
Posterior tibial slope $\left(^{\circ}\right)$ & & & \\
Preoperative value & $9.5 \pm 3.1$ & $8.3 \pm 2.7$ & 0.117 \\
Postoperative value & $10.9 \pm 4.0$ & $7.6 \pm 3.2$ & 0.001 \\
Posterior tibial slope change $\left(^{\circ}\right)$ & $1.4 \pm 2.6$ & $-0.8 \pm 2.3$ & 0.001 \\
\hline
\end{tabular}

Values are given as the mean \pm standard deviation.

\subsection{Measurement with the 3D Reconstructed Model}

In the $3 \mathrm{D}$ reconstructed model, the posterior tibial slope, osteotomy inclination angle relative to the medial tibial plateau line in the sagittal plane, and the ratio between the anterior and posterior osteotomy opening gaps were measured. There was no significant difference in the preoperative posterior tibial slope between the groups (group $\mathrm{A}=9.7 \pm 2.9^{\circ}$, group $\mathrm{P}=8.7 \pm 2.7^{\circ}, p=0.143$ ). However, the postoperative posterior tibial slope (group $\mathrm{A}=10.7 \pm 3.0^{\circ}$, group $\mathrm{P}=7.7 \pm 2.7^{\circ}, p<0.001$ ) and change in posterior tibial slope before and after surgery (group $\mathrm{A}=1.0 \pm 0.8^{\circ}$, group $\mathrm{P}=-0.9 \pm 0.8^{\circ}, p<0.001$ ) differed significantly between the groups (Table 4). A significant difference was also found in the comparison of the posterior tibial slope measured with the 3D reconstructed model 
before and after surgery for all patients (preoperative value $=9.4 \pm 2.9^{\circ}$, postoperative value $=9.9 \pm 3.2^{\circ}, p=0.001$ ). When comparing the preoperative and postoperative values within each group, there were significant differences in both groups A and P (group A: preoperative value $=9.7 \pm 2.9^{\circ}$, postoperative value $=10.7 \pm 3.0^{\circ}, p<0.001$; group $\mathrm{P}$ : preoperative value $=8.7 \pm 2.7^{\circ}$, postoperative value $\left.=7.7 \pm 2.7^{\circ}, p<0.001\right)$ (Table 3 ). The intraclass correlation coefficients for interobserver reliability using the $3 \mathrm{D}$ reconstructed model were 0.928 ( $95 \%$ CI, 0.888 0.954) for the preoperative posterior tibial slope and 0.937 (95\% CI, 0.902 0.960) for the postoperative posterior tibial slope. The mean osteotomy inclination angle in the sagittal plane was $4.9 \pm 3.2$ (range, $0.5^{\circ}$ to $15.0^{\circ}$ ) in group $\mathrm{A}$, and $-3.1 \pm 2.5$ (range, $-0.2^{\circ}$ to $\left.-10.6^{\circ}\right)$ in group $\mathrm{P}(p<0.001)$. The anterior opening gap was $8.2 \pm 2.2 \mathrm{~mm}$ (range, $4.2-14.5$ ), the posterior opening gap was $11.8 \pm 2.6 \mathrm{~mm}$ (range, 7.2-19.6), and the gap ratio was $69.7 \pm 8.4 \%$ in group A. The anterior opening gap was $8.3 \pm 1.4 \mathrm{~mm}$ (range, 5.4-10.4), the posterior opening gap was $12.6 \pm 2.4 \mathrm{~mm}$ (range, 8.1-17.0), and the gap ratio was $66.3 \pm 9.5 \%$ in group P. The gap ratio did not differ significantly between the groups $(p=0.118)$ (Table 4$)$. The intraclass correlation coefficients for the osteotomy inclination angle in the sagittal plane and the osteotomy opening gaps are shown in appendix 1. The statistical power assessed with $\mathrm{G}^{*}$ Power [30] was 99.2\% regarding the postoperative posterior tibial slope.

Table 3. Comparison between preoperative and postoperative measurement of posterior tibial slope on the plain radiograph and three-dimensional model.

\begin{tabular}{cccc}
\hline Variable & Preoperative PTS & Postoperative PTS & $p$-Value \\
\hline Plain radiography & & & \\
All patients $\left(^{\circ}\right)$ & $9.1 \pm 3.1$ & $9.8 \pm 4.1$ & 0.008 \\
group A $\left(^{\circ}\right)$ & $9.5 \pm 3.1$ & $10.9 \pm 4.0$ & $<0.001$ \\
group P $\left(^{\circ}\right)$ & $8.3 \pm 2.7$ & $7.6 \pm 3.2$ & 0.137 \\
Three-dimensional model & & & \\
All patients $\left(^{\circ}\right)$ & $9.4 \pm 2.9$ & $9.9 \pm 3.2$ & 0.001 \\
group A $\left(^{\circ}\right)$ & $9.7 \pm 2.9$ & $10.7 \pm 3.0$ & $<0.001$ \\
group P $\left(^{\circ}\right)$ & $8.7 \pm 2.7$ & $7.7 \pm 2.7$ & $<0.001$ \\
\hline
\end{tabular}

Values are given as the mean \pm standard deviation. PTS = Posterior tibial slope.

Table 4. Preoperative and postoperative measurement in the three-dimensional model.

\begin{tabular}{cccc}
\hline Variable & group A $(\boldsymbol{n = 5 8 )}$ & group P $(\boldsymbol{n = 2 2 )}$ & $p$-Value \\
\hline Posterior tibial slope $\left(^{\circ}\right)$ & & & \\
Preoperative value & $9.7 \pm 2.9$ & $8.7 \pm 2.7$ & 0.143 \\
Postoperative value & $10.7 \pm 3.0$ & $7.7 \pm 2.7$ & $<0.001$ \\
Posterior tibial slope change $\left(^{\circ}\right)$ & $1.0 \pm 0.8$ & $-0.9 \pm 0.8$ & $<0.001$ \\
Inclination angle $\left({ }^{\circ}\right)$ & $4.9 \pm 3.2$ & $-3.1 \pm 2.5$ & $<0.001$ \\
Gap ratio $(\%)$ & $69.7 \pm 8.4$ & $66.3 \pm 9.5$ & 0.118 \\
Anterior opening gap $(\mathrm{mm})$ & $8.2 \pm 2.2$ & $8.3 \pm 1.4$ & 0.916 \\
Posterior opening gap $(\mathrm{mm})$ & $11.8 \pm 2.6$ & $12.6 \pm 2.4$ & 0.185 \\
\hline
\end{tabular}

Values are given as the mean \pm standard deviation.

According to the Pearson correlation analysis, there was a significant correlation between two parameters, namely osteotomy inclination angle in sagittal plane and opening gap ratio and posterior tibial slope change. The Pearson correlation coefficient $(\mathrm{r})$ was $0.875(p<0.001)$ for the osteotomy inclination angle (Figure 5) and $0.233(p=0.038)$ for the gap ratio (Table 5). According to the subsequent multivariate regression analysis to identify significant independent variables affecting posterior tibial slope change, the only significant factor among the variables was the osteotomy inclination angle in the sagittal plane $(\beta$ coefficient $=0.216, p<0.001)($ Table 6$)$. 


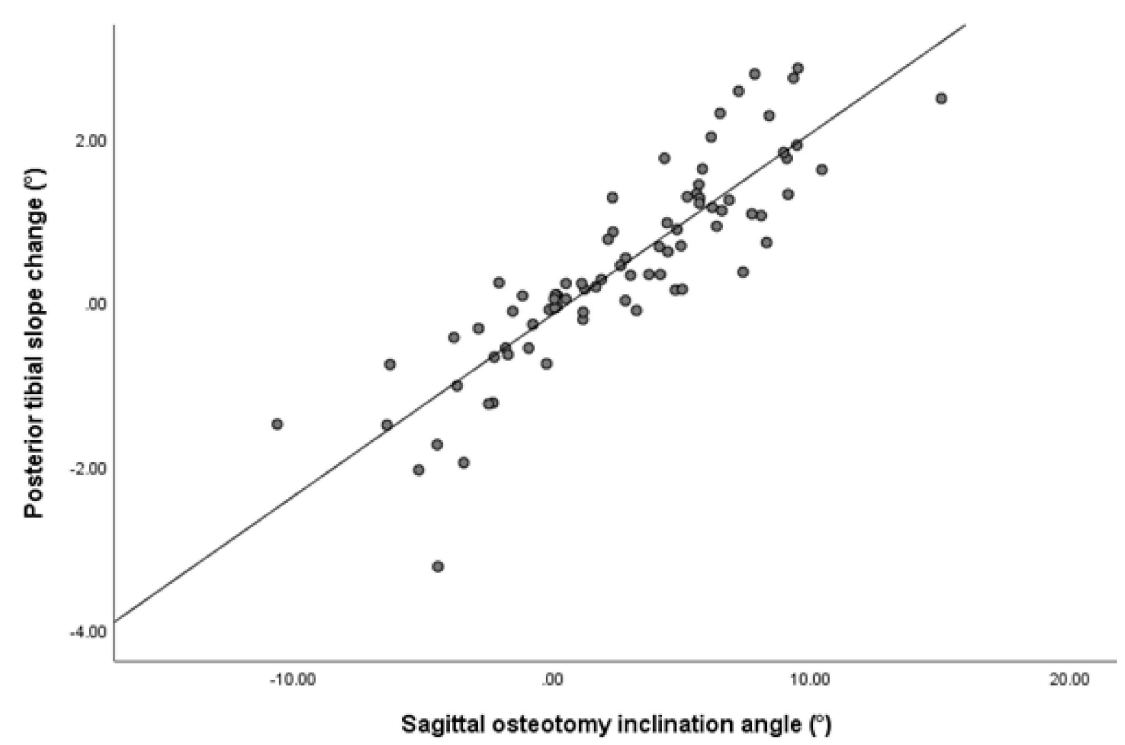

Figure 5. Scatter plot of Pearson correlation analysis showing the correlation between sagittal osteotomy inclination angle and the posterior tibial slope change. The sagittal osteotomy inclination angle has a significantly positive correlation with the posterior tibial slope change $(r=0.875, p<0.001)$.

Table 5. Correlation between variables and change in posterior tibial slope.

\begin{tabular}{ccc}
\hline \multirow{2}{*}{ Variable } & \multicolumn{2}{c}{ Posterior Tibial Slope Change } \\
\cline { 2 - 3 } & Pearson Correlation Coefficient (r) & $\boldsymbol{p}$ Value \\
\hline Inclination angle & 0.875 & $<0.001$ \\
Gap ratio & 0.233 & 0.038 \\
Correction angle & -0.170 & 0.133 \\
\hline
\end{tabular}

Table 6. Multivariate regression analysis for change in posterior tibial slope.

\begin{tabular}{cccc}
\hline Variable & $\boldsymbol{\beta}$ Coefficient & Standard Error & $p$ Value \\
\hline Inclination angle & 0.216 & 0.014 & $<0.001$ \\
Gap ratio & 0.011 & 0.008 & 0.149 \\
Correction angle & -0.026 & 0.027 & 0.332 \\
\hline
\end{tabular}

\section{Discussion}

The posterior tibial slope changes after MOWHTO, but little is known about the effect of the osteotomy inclination angle in the sagittal plane on the change in the posterior tibial slope. To investigate this effect, changes in the posterior tibial slope before and after surgery were measured, and the tendency and extent of the change in the posterior tibial slope were assessed by comparing two groups: anterior and posterior osteotomy inclination in the sagittal plane. According to the principal findings of this study, although the mean gap ratio between the anterior and posterior openings was maintained at an appropriate value, approximately 2:3, as recommended in a previous study [17], there was a significant difference in the posterior tibial slope before and after MOWHTO. In addition, the osteotomy inclination angle in the sagittal plane had a significant effect on the change in the posterior tibial slope. The anterior osteotomy inclination angle with the forward part of the osteotomy plane heading downward with respect to the medial tibial plateau line led to an increase in the postoperative posterior tibial slope. In contrast, the posterior osteotomy inclination angle led to a decrease in the postoperative posterior tibial slope.

Previous studies have noted that the posterior tibial slope changes after MOWHTO [12,18]. A recent meta-analysis also reported that posterior tibial slope increased by $2.02^{\circ}$ after 
MOWTHO [16]. Maintaining the posterior tibial slope before and after surgery is important to avoid unpredictable adverse effects. An increase in the posterior tibial slope could cause anterior translation of the tibia and increase the load on the anterior cruciate ligament [11,12]. An increased posterior tibial slope could also cause a redistribution of pressure into the posterior tibia in the anterior cruciate ligament-deficient knee, causing degenerative changes in articular cartilage [13] and an increase of vertical vector force onto the patellofemoral joint, which could lead to osteoarthritis of the patellofemoral joint $[14,15]$. In addition to the knee joint, it has been reported that the tibial slope also has important biomechanical implications on the ankle joint [31]. For these reasons, it is important to maintain the posterior tibial slope after MOWHTO, but there are practical difficulties in maintaining the posterior tibial slope during surgery because the proximal tibia is triangular, and osteotomy is performed from the anteromedial aspect without seeing the overall shape of the tibia. To avoid changes in the posterior tibial slope after MOWHTO, maintaining a constant ratio of the osteotomy opening gap between the anterior and posterior cortices has been emphasized [17,18]. Noyes et al. [18] reported that to maintain the original posterior tibial slope, the anterior gap should be one half of the posterior gap, and Song et al. [17] demonstrated that the normal posterior tibial slope can be unchanged if the anterior opening gap is approximately $67 \%$ of the posterior opening gap. To maintain the opening gap ratio, various factors leading to postoperative changes in the posterior tibial slope should be considered during surgery, such as incomplete posterior osteotomy, posterolateral hinge position, and an anteriorly placed plate affecting the gap ratio between the anterior and posterior cortices [26,32-34]. However, the results of this study showed that, although the mean opening gap ratio was maintained at an appropriate value, approximately 2:3 [17], the posterior tibial slope could change after MOWHTO. According to the comparison of posterior tibial slopes before and after surgery measured with $3 \mathrm{D}$ reconstructed models for all patients, there was a significant increase in the posterior tibial slope after surgery (preoperative value $=9.4 \pm 2.9^{\circ}$, postoperative value $=9.9 \pm 3.2^{\circ}$, $p=0.001$ ). Consequently, it is necessary to identify additional factors affecting the change in the posterior tibial slope after MOWHTO.

According to the results of this study, another factor affecting the change in the posterior tibial slope was the inclination angle of osteotomy in the sagittal plane. Few studies have dealt with the osteotomy inclination angle in relation to the posterior tibial slope $[19,35]$. A previous study recommended an osteotomy line parallel to the joint line to avoid inadvertent alteration of the posterior tibial slope [35]. Lee et al. [19] investigated the inclination angle of osteotomy in the sagittal plane and noted that only $12.9 \%$ of cases were conducted parallel to the medial joint line and $87.1 \%$ of osteotomy lines were anteriorly inclined. Anterior inclination of osteotomy in the sagittal plane was reported to increase the posterior tibial slope. In addition to these previous studies, our study comprehensively assessed how anterior or posterior inclination of the sagittal osteotomy line affects the change in the posterior tibial slope after MOWHTO, and the correlation between the osteotomy inclination angle and postoperative posterior tibial slope angle. In our study, $72.5 \%$ of patients had anteriorly inclined osteotomy, while $27.5 \%$ of patients had posteriorly inclined osteotomy. Similar to a previous study [19], the majority of osteotomy lines in the sagittal plane were anteriorly inclined. According to the data measured with $3 \mathrm{D}$ reconstructed models, the preoperative posterior tibial slope was $9.7 \pm 2.9^{\circ}$ and postoperative posterior tibial slope was $10.7 \pm 3.0^{\circ}$ in group A. The preoperative posterior tibial slope was $8.7 \pm 2.7^{\circ}$ and the postoperative posterior tibial slope was $7.7 \pm 2.7^{\circ}$ in group P. The mean osteotomy inclination angle in the sagittal plane was $4.9 \pm 3.2$ in group A and $-3.1 \pm 2.5$ in group P. Although there was no significant difference in the osteotomy opening gap ratio between the groups, the postoperative posterior tibial slope changed in different direction depending on the difference in the inclination angle in sagittal plane. When the osteotomy line was inclined anteriorly with respect to the medial tibial plateau line, the postoperative posterior tibial slope tended to increase. In contrast, when the osteotomy line was inclined posteriorly, the postoperative posterior 
tibial slope tended to decrease. Both the Pearson correlation $(\mathrm{r}=0.875, p<0.001)$ and multiple regression analyses ( $\beta$ coefficient $=0.216, p<0.001$ ) also showed a significant association between the osteotomy inclination angle in the sagittal plane and change in posterior tibial slope. Accordingly, to avoid inadvertent effects on the kinematics of the knee joint, close attention needs to be paid to maintaining the sagittal osteotomy line parallel to the medial joint line during MOWHTO. In addition, in cases where the treatment effect is expected by intentionally adjusting the posterior tibial slope, such as for osteoarthritis with anteroposterior instability, the posterior tibial slope could be controlled by varying the inclination angle of the osteotomy in the sagittal plane [12].

Another notable finding in this study was that measurements with 3D reconstructed models were more reliable than measurement with plain radiographs. When measured with plain radiographs, the intraclass correlation coefficients for interobserver reliability were 0.748 (95\% CI, 0.607 0.838) for the preoperative posterior tibial slope and 0.759 ( $95 \%$ CI, 0.625 0.846) for the postoperative posterior tibial slope. However, when measured with 3D reconstructed models, the intraclass correlation coefficients for interobserver reliability were 0.928 ( $95 \% \mathrm{CI}, 0.888 \sim 0.954$ ) for the preoperative posterior tibial slope and 0.937 (95\% CI, 0.902 0.960) for the postoperative posterior tibial slope. In comparing the preoperative and postoperative posterior tibial slopes in group $\mathrm{P}$, measured values with plain radiographs did not differ significantly, but measured values with $3 \mathrm{D}$ reconstructed models differed significantly. There is still no established method for measuring the posterior tibial slope. A more reliable and generally agreeable method uses the medial tibial plateau line and anatomical axis of the proximal tibial shaft or tibial cortex line [36]. However, the measurement method using plain radiographs has disadvantages such as poor reproducibility due to the difficulties in obtaining true lateral images of the tibia caused by rotation and distinguishing between the medial and lateral tibial plateau because they overlap [37]. For these reasons, CT scans and magnetic resonance imaging have been recently used for measurement of the posterior tibial slope. In this study, measurements were performed with $3 \mathrm{D}$ reconstructed models using the method presented in previous studies $[25,26]$. The results of our study also demonstrated that measurements with 3D reconstructed models obtain more consistent values with higher interobserver reliability.

This study had several limitations. Firstly, this study was based on retrospectively collected data. In order to obtain a more solid conclusion, a prospective study design by means of a cadaver study or finite elements is needed. Secondly, a preoperative CT scan was not performed. The preoperative $3 \mathrm{D}$ tibia model was reconstructed by digitally removing the osteotomy gap from the postoperative $3 \mathrm{D}$ tibia model using a lateral hinge as a rotation axis. Although the preoperative 3D tibia model was restored as close to the actual preoperative tibia as possible, there could be a difference with the actual preoperative tibia. Thirdly, the number of patients in each study group was different. The majority of osteotomies in the sagittal plane were anteriorly inclined. Therefore, the number of patients in group A was larger than in group P. The relatively small number of patients could decrease statistical power. However, the calculated statistical power was $99.2 \%$. Thus, the small number of patients in group P was not considered a serious problem. Fourthly, there was a statistically significant difference between the preoperative and postoperative posterior tibial slopes in all patients and in each group. There was also a statistically significant difference in postoperative posterior tibial slopes between the groups. However, as with the minimal clinically important difference, it has not been revealed how much the difference in posterior tibial slope actually affects the clinical outcomes. Accordingly, to determine the clinical effect of the change in posterior tibial slope on functional outcomes, a more in-depth study with long-term clinical follow-up results is needed.

\section{Conclusions}

Although the osteotomy gap ratio between the anterior and posterior gaps was kept at an appropriate value, the posterior tibial slope changed after MOWHTO according to the osteotomy inclination angle in the sagittal plane. The postoperative posterior tibial 
slope tended to increase when the osteotomy line was inclined anteriorly with respect to the medial tibial plateau line but decreased when the osteotomy line was inclined posteriorly. To avoid inadvertent effects on the kinematics of the knee joint, close attention needs to be paid to maintaining the sagittal osteotomy line parallel to the medial joint line during MOWHTO. This study would be helpful to understanding the effect of the osteotomy inclination angle in the sagittal plane on the change in the posterior tibial slope after MOWHTO.

Author Contributions: Conceptualization, C.H.C., S.-H.K., S.-J.K., and M.J.; methodology, J.H.C. and M.J.; investigation, J.H.C. and M.J.; data curation, J.H.C. and S.-K.L.; writing—original draft preparation, J.H.C. and M.J.; writing-review \& editing, M.J. All authors have read and agreed to the published version of the manuscript.

Funding: This study was supported by a faculty research grant of Yonsei University College of Medicine for (6-2020-0095).

Institutional Review Board Statement: The study was approved by the Institutional Review Board of Severance Hospital, Yonsei University College of Medicine (4-2019-0800).

Informed Consent Statement: Patient consent was waived due to retrospective type of the study.

Data Availability Statement: The data presented in this study may be available on request from the corresponding author. The data are not publicly available due to privacy and ethical considerations.

Acknowledgments: We wish to thank two orthopedic surgeons, Jongtaek Oh and Hojun Choi, for their assistance in the measurement of parameters.

Conflicts of Interest: The authors declare no conflict of interest.

\section{Appendix A}

Table A1. Intraclass correlation coefficient.

\begin{tabular}{ccc}
\hline Variable & ICC & 95\% Confidence Interval \\
\hline Hip-Knee-Ankle angle & & \\
Preoperative & 0.907 & $0.868-0.936$ \\
Postoperative & 0.916 & $0.881-0.943$ \\
Weight-bearing line ratio & & \\
Preoperative & 0.923 & $0.892-0.948$ \\
Postoperative & 0.927 & $0.896-0.950$ \\
Osteotomy inclination angle & 0.942 & $0.918-0.960$ \\
Osteotomy opening gap & & \\
Anterior opening gap & 0.936 & $0.910-0.956$ \\
Posterior opening gap & 0.930 & $0.902-0.952$ \\
\hline
\end{tabular}

ICC = Intraclass correlation coefficient.

\section{References}

1. Asada, S.; Akagi, M.; Mori, S.; Matsushita, T.; Hashimoto, K.; Hamanishi, C. Increase in posterior tibial slope would result in correction loss in frontal plane after medial open-wedge high tibial osteotomy. Knee Surg. Sports Traumatol. Arthrosc. 2012, 20, 571-578. [CrossRef]

2. Aglietti, P.; Rinonapoli, E.; Stringa, G.; Taviani, A. Tibial osteotomy for the varus osteoarthritic knee. Clin. Orthop. Relat. Res. 1983, 176, 239-251. [CrossRef]

3. Lobenhoffer, P.; Agneskirchner, J.D. Improvements in surgical technique of valgus high tibial osteotomy. Knee Surg. Sports Traumatol. Arthrosc. 2003, 11, 132-138. [CrossRef] [PubMed]

4. Coventry, M.B. Osteotomy of the upper portion of the tibia for degenerative arthritis of the knee. A preliminary report. J. Bone Joint Surg. Am. 1965, 47, 984-990. [CrossRef] [PubMed]

5. Akizuki, S.; Shibakawa, A.; Takizawa, T.; Yamazaki, I.; Horiuchi, H. The long-term outcome of high tibial osteotomy: A ten- to 20-year follow-up. J. Bone Joint Surg. Br. 2008, 90, 592-596. [CrossRef] [PubMed]

6. Koshino, T.; Yoshida, T.; Ara, Y.; Saito, I.; Saito, T. Fifteen to twenty-eight years' follow-up results of high tibial valgus osteotomy for osteoarthritic knee. Knee 2004, 11, 439-444. [CrossRef] [PubMed] 
7. Kyung, H.-S.; Lee, B.-J.; Kim, J.-W.; Yoon, S.-D. Biplanar open wedge high tibial osteotomy in the medial compartment osteoarthritis of the knee joint: Comparison between the aescula and tomofix plate. Clin. Orthop. Surg. 2015, 7, 185-190. [CrossRef]

8. Lee, S.S.; Lee, H.I.; Cho, S.T.; Cho, J.H. Comparison of the outcomes between two different target points after open wedge high tibial osteotomy: The fujisawa point versus the lateral tibial spine. Knee 2020, 27, 915-922. [CrossRef]

9. Matsushita, T.; Watanabe, S.; Araki, D.; Nagai, K.; Hoshino, Y.; Kanzaki, N.; Matsumoto, T.; Niikura, T.; Kuroda, R. Differences in preoperative planning for high-tibial osteotomy between the standing and supine positions. Knee Surg. Relat. Res. 2021, 33, 8. [CrossRef]

10. Na, Y.G.; Lee, B.K.; Choi, J.U.; Lee, B.H.; Sim, J.A. Change of joint-line convergence angle should be considered for accurate alignment correction in high tibial osteotomy. Knee Surg. Relat. Res. 2021, 33, 4. [CrossRef]

11. Kim, G.B.; Kim, K.I.; Song, S.J.; Lee, S.H. Increased posterior tibial slope after medial open-wedge high tibial osteotomy may result in degenerative changes in anterior cruciate ligament. J. Arthroplast. 2019, 34, 1922-1928. [CrossRef]

12. Giffin, J.R.; Vogrin, T.M.; Zantop, T.; Woo, S.L.; Harner, C.D. Effects of increasing tibial slope on the biomechanics of the knee. Am. J. Sports Med. 2004, 32, 376-382. [CrossRef]

13. Rodner, C.M.; Adams, D.J.; Diaz-Doran, V.; Tate, J.P.; Santangelo, S.A.; Mazzocca, A.D.; Arciero, R.A. Medial opening wedge tibial osteotomy and the sagittal plane: The effect of increasing tibial slope on tibiofemoral contact pressure. Am. J. Sports Med. 2006, 34, 1431-1441. [CrossRef] [PubMed]

14. El-Azab, H.; Halawa, A.; Anetzberger, H.; Imhoff, A.B.; Hinterwimmer, S. The effect of closed- and open-wedge high tibial osteotomy on tibial slope: A retrospective radiological review of 120 cases. J. Bone Joint Surg. Br. 2008, 90, 1193-1197. [CrossRef] [PubMed]

15. Tseng, T.H.; Tsai, Y.C.; Lin, K.Y.; Kuo, Y.K.; Wang, J.H. The correlation of sagittal osteotomy inclination and the anteroposterior translation in medial open-wedge high tibial osteotomy-one of the causes affecting the patellofemoral joint? Int. Orthop. 2019, 43, 605-610. [CrossRef] [PubMed]

16. Nha, K.W.; Kim, H.J.; Ahn, H.S.; Lee, D.H. Change in posterior tibial slope after open-wedge and closed-wedge high tibial osteotomy: A meta-analysis. Am. J. Sports Med. 2016, 44, 3006-3013. [CrossRef]

17. Song, E.K.; Seon, J.K.; Park, S.J. How to avoid unintended increase of posterior slope in navigation-assisted open-wedge high tibial osteotomy. Orthopedics 2007, 30, S127-S131.

18. Noyes, F.R.; Goebel, S.X.; West, J. Opening wedge tibial osteotomy: The 3-triangle method to correct axial alignment and tibial slope. Am. J. Sports Med. 2005, 33, 378-387. [CrossRef] [PubMed]

19. Lee, S.Y.; Lim, H.C.; Bae, J.H.; Kim, J.G.; Yun, S.H.; Yang, J.H.; Yoon, J.R. Sagittal osteotomy inclination in medial open-wedge high tibial osteotomy. Knee Surg. Sports Traumatol. Arthrosc. 2017, 25, 823-831. [CrossRef] [PubMed]

20. Brittberg, M.; Winalski, C.S. Evaluation of cartilage injuries and repair. J. Bone Joint Surg. Am. 2003, 85 (Suppl. S2), 58-69. [CrossRef]

21. Miniaci, A.; Ballmer, F.T.; Ballmer, P.M.; Jakob, R.P. Proximal tibial osteotomy. A new fixation device. Clin. Orthop. Relat. Res. 1989, 246, 250-259.

22. Fujisawa, Y.; Masuhara, K.; Shiomi, S. The effect of high tibial osteotomy on osteoarthritis of the knee. An arthroscopic study of 54 knee joints. Orthop. Clin. N. Am. 1979, 10, 585-608. [CrossRef]

23. Lee, Y.S.; Park, S.J.; Shin, V.I.; Lee, J.H.; Kim, Y.H.; Song, E.K. Achievement of targeted posterior slope in the medial opening wedge high tibial osteotomy: A mathematical approach. Ann. Biomed. Eng. 2010, 38, 583-593. [CrossRef] [PubMed]

24. Kim, S.H.; Kim, S.J.; Choi, C.H.; Kim, D.; Jung, M. Optimal condition to create femoral tunnel considering combined influence of knee flexion and transverse drill angle in anatomical single-bundle acl reconstruction using medial portal technique: $3 \mathrm{~d}$ simulation study. Biomed. Res. Int. 2018, 2018, 2643247. [CrossRef] [PubMed]

25. Lee, B.H.; Ha, C.W.; Moon, S.W.; Chang, M.; Kim, H.Y.; Park, S.H.; Wang, J.H. Three-dimensional relationships between secondary changes and selective osteotomy parameters for biplane medial open-wedge high tibial osteotomy. Knee 2017, 24, 362-371. [CrossRef]

26. Moon, S.W.; Park, S.H.; Lee, B.H.; Oh, M.; Chang, M.; Ahn, J.H.; Wang, J.H. The effect of hinge position on posterior tibial slope in medial open-wedge high tibial osteotomy. Arthroscopy 2015, 31, 1128-1133. [CrossRef] [PubMed]

27. Ogawa, H.; Matsumoto, K.; Ogawa, T.; Takeuchi, K.; Akiyama, H. Effect of wedge insertion angle on posterior tibial slope in medial opening wedge high tibial osteotomy. Orthop. J. Sports Med. 2016, 4, 2325967116630748. [CrossRef]

28. Yoon, T.H.; Choi, C.H.; Kim, S.J.; Kim, S.H.; Kim, N.H.; Jung, M. Effect of medial open-wedge high tibial osteotomy on the patellofemoral joint according to postoperative realignment. Am. J. Sports Med. 2019, 47, 1863-1873. [CrossRef] [PubMed]

29. Akamatsu, Y.; Sotozawa, M.; Kobayashi, H.; Kusayama, Y.; Kumagai, K.; Saito, T. Usefulness of long tibial axis to measure medial tibial slope for opening wedge high tibial osteotomy. Knee Surg. Sports Traumatol. Arthrosc. 2016, 24, 3661-3667. [CrossRef]

30. Faul, F.; Erdfelder, E.; Buchner, A.; Lang, A.G. Statistical power analyses using g*power 3.1: Tests for correlation and regression analyses. Behav. Res. Methods 2009, 41, 1149-1160. [CrossRef]

31. Usuelli, F.G.; Maccario, C.; Indino, C.; Manzi, L.; Gross, C.E. Tibial slope in total ankle arthroplasty: Anterior or lateral approach. Foot Ankle Surg.Off. J. Eur. Soc. Foot Ankle Surg. 2017, 23, 84-88. [CrossRef]

32. Wang, J.H.; Bae, J.H.; Lim, H.C.; Shon, W.Y.; Kim, C.W.; Cho, J.W. Medial open wedge high tibial osteotomy: The effect of the cortical hinge on posterior tibial slope. Am. J. Sports Med. 2009, 37, 2411-2418. [CrossRef] 
33. Rubino, L.J.; Schoderbek, R.J.; Golish, S.R.; Baumfeld, J.; Miller, M.D. The effect of plate position and size on tibial slope in high tibial osteotomy: A cadaveric study. J. Knee Surg. 2008, 21, 75-79. [CrossRef] [PubMed]

34. Marti, C.B.; Gautier, E.; Wachtl, S.W.; Jakob, R.P. Accuracy of frontal and sagittal plane correction in open-wedge high tibial osteotomy. Arthroscopy 2004, 20, 366-372. [CrossRef] [PubMed]

35. Miller, B.S.; Downie, B.; McDonough, E.B.; Wojtys, E.M. Complications after medial opening wedge high tibial osteotomy. Arthroscopy 2009, 25, 639-646. [CrossRef] [PubMed]

36. Cullu, E.; Aydogdu, S.; Alparslan, B.; Sur, H. Tibial slope changes following dome-type high tibial osteotomy. Knee Surg. Sports Traumatol. Arthrosc. 2005, 13, 38-43. [CrossRef]

37. Ho, J.P.Y.; Merican, A.M.; Hashim, M.S.; Abbas, A.A.; Chan, C.K.; Mohamad, J.A. Three-dimensional computed tomography analysis of the posterior tibial slope in 100 knees. J. Arthroplast. 2017, 32, 3176-3183. [CrossRef] 